\title{
Topical nepafenac for prevention of post-cataract surgery macular edema in diabetic patients: patient selection and perspectives
}

This article was published in the following Dove Press journal:

Clinical Ophthalmology

\author{
Bora Yüksel \\ Ömer Karti \\ Tuncay Kusbeci \\ Department of Ophthalmology, \\ Bozyaka Training and Research \\ Hospital, İzmir, Turkey
}

Correspondence: Bora Yüksel

Department of Ophthalmology, Bozyaka Training and Research Hospital, Saim Çıkrıkcı Caddesi No: 59, Bozyaka, İzmir 35I70, Turkey

Tel +905324429844

Fax +90 232 26I 4444

Email drborayuksel@gmail.com

\begin{abstract}
Since its first description, the prevention of pseudophakic cystoid macular edema (PCME) continues to pose challenges for ophthalmologists. Recent evidence suggests that prophylaxis is unnecessary in patients without risk factors. Diabetes mellitus is generally considered as a risk factor for the development of PCME after cataract surgery since it causes breakdown of the blood-retinal barrier. Diabetic retinopathy (DR) increases the risk even further. Therefore, prophylactic nonsteroidal anti-inflammatory drugs (NSAIDs) should be considered in diabetic patients, especially if they have DR. NSAIDs block the cyclooxygenase enzymes responsible for prostaglandin production and reduce the incidence of PCME after cataract surgery. Nepafenac seems superior to other NSAIDs in terms of ocular penetration allowing higher and sustained therapeutic levels in retina and choroid. Topical steroids are less effective and may cause intraocular pressure increase limiting their long-term use. Nepafenac is cost effective, when the burden of PCME prevention is compared with the burden of treatment. Prevention is much cheaper and less harmful than invasive treatments like periocular or intravitreal injections. Overall, both nepafenac $0.1 \%$ and nepafenac $0.3 \%$ are well tolerated. They should be used carefully in patients with compromised corneas such as those with severe dry eye or penetrating grafts. If otherwise healthy cataract patients have $\geq 2$ risk factors, like PCME in the other eye or posterior capsule rupture during surgery, treatment should be considered. Once-daily nepafenac $0.3 \%$ dosing may improve postoperative outcomes through increased patient compliance and may reduce treatment burden further. Every patient should be assessed in terms of risks/benefits of the treatment, in individual basis, before cataract surgery.
\end{abstract}

Keywords: cataract surgery, macular edema, prevention, nepafenac, diabetic patients

\section{Introduction}

Cystoid macular edema (CME) following cataract surgery, also known as Irvine-Gass syndrome, is a well-known cause of poor vision following uneventful cataract surgery. ${ }^{1-3}$ The incidence of clinical (symptomatic) pseudophakic cystoid macular edema (PCME) has been greatly reduced because of the advances in surgical techniques (approximately $0.1 \%-2.3 \%$ ) including phacoemulsification and small-incision cataract surgery. ${ }^{1,4}$ A large retrospective study including 81,984 eyes revealed that the incidence of PCME in eyes without operative complications, diabetes, or risk factors was $1.17 \% .^{5}$ The incidence of clinically significant PCME peaks at approximately 5 (4-12) weeks in a healthy population. ${ }^{4}$ The condition is usually self-limiting, and the resolution of symptoms usually occurs within 3-12 months; it may occasionally persist and lead to permanent vision loss. ${ }^{1}$ 
Majority of patients remain asymptomatic without active inflammation on fundus examination and optical coherence tomography (OCT). However, subclinical PCME is detected in almost $30 \%$ of patients with postsurgical fluorescein angiography and a further $11 \%-41 \%$ of patients with OCT. ${ }^{6}$ The incidence may be as high as $20 \%$ when cataract extraction is complicated by posterior capsule rupture with vitreous loss or severe iris trauma. ${ }^{5,7}$ Macular changes are more likely to occur following cataract surgery in diabetic patients, especially those with preexisting retinopathies compared to nondiabetic patients. ${ }^{4}$ The rate of development of macular edema (ME) in diabetic population (with or without diabetic retinopathy $[\mathrm{DR}]$ ) varies across studies, ranging from $31 \%$ to $81 \%$ at various time points following cataract extraction. ${ }^{2}$ Chu et al reported an increased relative risk (RR) in patients with diabetes ( $R R, 1.80)$. The risk was higher in the presence of any DR (RR, 6.23). ${ }^{5}$

The pathogenesis of PCME is likely multifactorial, ultimately leading to the breakdown of the blood-aqueous and blood-retinal barrier and cystic accumulation of extracellular intraretinal fluid. ${ }^{1,8}$ During the surgery, inflammatory mediators are released from lens epithelial cells and uveal tissue in the anterior segment. If mediators diffuse to the vitreous and retina, the inflammatory response will cause local vasodilatation and disruption of the blood-retinal barrier. Increased vascular permeability may lead to fluid accumulation in the inner nuclear and outer plexiform layers of the retina, and CME will develop. ${ }^{6}$ Most research studies have focused on the role of prostaglandins. Recent studies, however, have shown the importance of other inflammatory mediators such as vascular endothelial growth factor (VEGF) and various cytokines. ${ }^{9}$ In diabetic macular edema (DME), ME is induced by hyperglycemia-induced oxidative stress, deposition of advanced glycation end products (AGEs), impaired blood flow, hypoxia, pericyte loss, endothelial cell loss, downregulation of glial cell-derived neurotrophic factor, blood-retinal barrier dysfunction, and chronic subclinical inflammation. ${ }^{2,4}$ An increased intraocular concentration of proinflammatory interleukins IL-6, IL-8, and IL-12 has been reported in patients with diabetes compared to patients without diabetes. ${ }^{10}$

\section{Predisposing factors}

Although PCME can occur in healthy eyes with no surgical complications, risk factors increase the likelihood of it occurring. Patient factors predisposing to PCME include PCME in the contralateral eye, African-American origin, and anything that may disrupt the blood-retinal barrier such as diabetes mellitus (DM), uveitis, retinal vein occlusion, retinal degeneration, macular degeneration, radiation retinopathy, epiretinal membranes, choroidal tumors, prostaglandin analog use, and aging. ${ }^{1,8,10}$ Surgical factors include inflammationinducing complications such as retained lens material, posterior capsule rupture, vitreous loss and vitreomacular traction, and excessive intraoperative manipulations such as mechanical pupil stretch or iris prolapse during surgery, the presence of an anterior chamber intraocular lens, filtering or other glaucoma operations, and intraocular surgeries. ${ }^{1,12}$ Do et al reported that the transient corneal edema (TCE) detected 1 week after surgery was a significant risk factor for the development of PCME (odds ratio [OR], 6.71; $p=0.003$ ) at 5 weeks. They also found that DM was significantly associated with TCE (OR, 4.04; $p=0.011)$ and PCME (OR, 4.58; $p=0.007)$. The relationship between TCE and development of PCME suggests that TCE and PCME may partly share the same etiologies of inflammation, breakdown of the bloodaqueous and blood-retinal barriers, and increased vascular permeability. ${ }^{13}$

\section{PCME definition on OCT}

The diagnosis of CME was previously made with fluorescein angiography after the patient became symptomatic; however, the emergence of OCT has provided a quick and noninvasive means of diagnosis. ${ }^{1}$ The clinical definition of PCME on OCT varies among studies like following: a $\geq 40 \%$ increase in central foveal thickness (CFT) on OCT and a $\geq 20 \%$ decrease in macular sensitivity, ${ }^{14} \mathrm{a}>40 \mu \mathrm{m}$ increase in CFT from baseline, ${ }^{15} \mathrm{a} \geq 30 \%$ increase in CFT from baseline within 90 days following surgery, ${ }^{2,16}$ a visual acuity of $20 / 40$ or worse and spectral domain-OCT CFT $>320 \mu \mathrm{m},{ }^{11}$ a CFT increase $>10 \%$ from baseline, ${ }^{17} \mathrm{a} \geq 30 \%$ increase within 90 days after surgery, ${ }^{7}$ and a $35 \%$ increase in macular volume. ${ }^{8}$ This variation makes the comparison of different studies difficult.

\section{Differentiation of PCME from DME}

Sometimes, it is difficult to differentiate DME from PCME after cataract surgery in diabetic patients. However, in order to properly treat these eyes, it is crucial to define ME appropriately. Pathogenesis of DME involves chronic hyperglycemia associated with AGEs, activation of protein-kinase C, polyol, and the hexosamine pathways, leading to the breakdown of cell junctions, pericyte loss, and inflammation, whereas PCME is induced by an acute and local inflammatory reaction leading to release of proinflammatory cytokines, prostaglandins, proteases, and complement. Such an acute inflammation 
may lead to subretinal fluid (SRF) due to dysfunction of the retinal pigment epithelial cells and acute breakdown of the inner and/or outer blood-retinal barrier. ${ }^{4}$ Munk et al described OCT criteria to differentiate PCME from DME. A higher $\mathrm{CFT} /$ retinal volume ratio, a thicker outer nuclear layer (ONL)/Henle's layer, solely inner nuclear layer (INL) cysts, absence of epiretinal membrane, intact hyperreflective outer retinal bands, and presence of SRF likely refer to PCME, whereas a higher ONL/INL thickness ratio parafoveally, the presence of microaneurysms, hard exudates, and microfoci, the presence of additional ganglion cell or retinal nerve fiber layer cysts, and the absence of SRF, mainly ONL cysts, and disruption of the photoreceptor layers are characteristic of DME. In contrast to the PCME eyes, the majority of DME eyes preserve foveal depression. ${ }^{4}$

\section{Increased risk in diabetic patients}

Patients with diabetes are at an increased risk of developing cataract compared with those without diabetes, and the rising global prevalence of diabetes further increases the risk of cataract development. Cataract development occurs at an earlier age in diabetics. ${ }^{7}$ Diabetic keratopathy occurs in $70 \%$ of the patients with DM. It may lead to recurrent erosions, decreased corneal sensitivity, delayed epithelial healing, epithelial and endothelial barrier dysfunctions, corneal edema, and infectious ulsers. ${ }^{18,19}$ Patients with diabetes, particularly those with DR, are at an increased risk of developing PCME. Do et al reported the incidence of PCME as $25 \%$ in diabetic patients. ${ }^{13}$ Chu et al reported an increased RR of ME after cataract surgery in patients with DR and that this RR rose proportionately with increasing severity of DR (DM without retinopathy 2.15 , very mild nonproliferative DR [NPDR] 3.50, severe NPDR 7.69, proliferative DR [PDR] 12.07, panretinal laser and stable PDR 10.63, any DR 7.27). ${ }^{5}$ The duration, severity, and type of diabetes, hardness of the lens, and $\mathrm{HbA}_{1 \mathrm{c}}$ are other contributing factors. ${ }^{14}$ Eyes with DME prior to cataract surgery are at higher risk of developing central-involved ME. ${ }^{20}$ It is important to monitor macular changes in patients with DM after cataract surgery. Clinically significant PCME usually develops within 4-12 weeks after surgery approximately peaking at $4-6$ weeks. ${ }^{7}$ There is no consensus in literature on the incidence of PCME in patients with DM.

A large retrospective database study including 139,759 patients revealed a higher incidence of PCME in diabetics (3.05\%) compared to nondiabetics (1.73\%). ${ }^{21}$ In Munk et al's study, the incidence of clinically significant PCME after uncomplicated cataract surgery was reported as $16.3 \%$ in patients with previous DME and in patients with DR, whose blood-retinal barrier has already been compromised before surgery. ${ }^{4}$ Recent studies using OCT have reported higher rates of PCME in patients with diabetes with or without retinopathy $(18.0 \%-28.6 \%)$ than in those without diabetes $(1 \%-5.5 \%){ }^{7}$ whereas Shorstein et al found no important association of PCME risk with DM (without regard to retinopathy). ${ }^{11}$

\section{Need for prophylaxis}

The prevention of Irvine-Gass syndrome continues to pose challenges for ophthalmologists, due to its ability to affect individuals without any obvious predisposing factors at variable times of onset postoperatively. ${ }^{1}$ Some studies demonstrated negligible benefit with nonsteroidal anti-inflammatory drug (NSAID) use after uncomplicated cataract surgery in patients without risk factors, ${ }^{22,23}$ whereas others support their use in the treatment and prophylaxis of PCME in uncomplicated cases. ${ }^{8}$ In subjects undergoing cataract surgery, and at low risk for PCME, the routine use of preoperative nepafenac was suggested necessary only to achieve a faster visual recovery. ${ }^{24}$ However, prophylactic therapy should be considered in those with high risk like diabetic patients especially those with DR due to the deficient blood-retinal barrier and advanced vascular changes. ${ }^{5}$ Options for prevention of ME after cataract surgery in patients with NPDR include preoperative treatment with steroids, intravitreal injections of anti-VEGFs, laser treatment, and topical NSAIDs. ${ }^{17}$ It seems more prudent to prevent PCME rather than treat it, since treatment usually involves more invasive methods like intravitreal NSAID, ${ }^{25}$ subtenon triamcinolone, ${ }^{26}$ or intravitreal dexamethasone implant injections. ${ }^{6}$ Boscia et al suggested that all diabetic patients undergoing cataract surgery should be treated with topical NSAIDs to prevent PCME. Intravitreal anti-VEGF drugs and steroids, combined with cataract surgery, may be considered in patients with preexisting DME. ${ }^{27}$

\section{General aspects of NSAIDs}

Given that topical NSAIDs block the cyclooxygenase (COX) enzymes responsible for prostaglandin production, they may also reduce the incidence, duration, and severity of $\mathrm{ME} .^{2}$ Several trials about PCME following cataract surgery showed positive effect of NSAIDs on postoperative ME. ${ }^{10,28,29}$ The topical use of $0.1 \%$ diclofenac in patients with DR, 4 times a day for 7 days before cataract surgery lasting 30 days, resulted in significantly lower intraocular levels of IL-12 and a lower increase in CFT compared to standard postoperative therapy with $0.1 \%$ topical dexamethasone 4 times a day for 30 days. ${ }^{10}$ 
In a recent report by the American Academy of Ophthalmology, NSAID therapy was found effective in reducing PCME and increasing the speed of visual recovery after surgery when compared directly with placebo or topical corticosteroid formulations with limited intraocular penetration. However, they concluded that the efficacy of NSAID use on long-term visual outcomes was unclear. ${ }^{30} \mathrm{~A}$ review by Lim et al revealed that topical NSAIDs (including ketorolac, diclofenac, nepafenac, indomethacin, bromfenac, flurbiprofen, and pranopfen) in combination with steroids were associated with a reduced risk of PCME; however, it was again unclear that this reduction has an impact on visual function after surgery. ${ }^{28}$ In a retrospective analysis including 89,731 eyes, the prevalence of PCME was found to be $1.3 \%$ with NSAIDs and $1.7 \%$ without them. The number needed to treat was 320 patients to prevent 1 case of PCME. NSAID use was associated with a lower incidence of PCME in patients without diabetes (RR, 0.68) and diabetics without retinopathy (RR, 0.51). Interestingly, NSAID use was not associated with a change in the incidence of PCME among patients with DR (RR, 1.06). ${ }^{29}$

Shields et al reported significant reductions in the incidence of clinical PCME with prophylactic use of topical NSAIDs, including ketorolac $0.4 \%$ or nepafenac $0.1 \%$, in conjunction with topical steroids. ${ }^{1}$ In Kessel et al's systematic review including randomized trials comparing topical steroids with topical NSAIDs in preventing PCME after phacoemulsification, a high-quality evidence was found indicating that topical NSAIDs are more effective than topical steroids in the prevention of PCME. ${ }^{31}$ In Shorstein et al's retrospective cohort study, postoperative topical prednisolone acetate (PA) alone or in combination with NSAIDs (diclofenac, flurbiprofen, and ketorolac tromethamine) was compared. Clinically significant PCME with a visual acuity of $\leq 20 / 40$ within 120 days after phacoemulsification was found in $0.73 \%$ among 16,070 surgeries. Compared with PA alone, the OR for PA + NSAID was $0.45 .{ }^{11}$ McCafferty et al reported the RR for PCME as 19.5 in contralateral PCME and 13.1 in DR. They compared topical Ilevro ${ }^{\circledR}$ (nepafenac $0.3 \%$ ) with placebo and found that topical nepafenac $0.3 \%$ reduces PCME in patients with preoperative risk factors compared to placebo but shows no benefit in patients without risk factors. ${ }^{8}$ Briefly, the efficacy of NSAIDs in the prevention of PCME is less apparent in general population including otherwise healthy subjects undergoing cataract surgery.

\section{Comparison of nepafenac with other NSAIDs}

After approval in 2005, topical Nevanac ${ }^{\circledR}$ (nepafenac 0.1\%; Alcon Research, Ltd., Fort Worth, TX, USA) ophthalmic suspension is widely prescribed to manage pain, inflammation, and ME after cataract surgery. ${ }^{17}$ Nepafenac $0.1 \%$ is also approved in Europe for the reduction in the risk of PCME associated with cataract surgery in diabetic patients. ${ }^{32}$ Nepafenac is a prodrug that rapidly penetrates the cornea and is deaminated to form the active metabolite, amfenac, by intraocular hydrolases within ciliary body epithelium, retina, and choroid. Nepafenac and amfenac are potent inhibitors of the COX enzyme isoforms (COX1, COX2). Nepafenac has a prolonged activity in the vascularized tissues of the eye. ${ }^{2}$ In a nonindustry-supported study, Hariprasad et al explained nepafenac's apparent advantage based on preclinical evidence as follows: Nepafenac exhibits enhanced corneal permeability relative to diclofenac, allowing for greater intraocular drug accumulation. The rate of hydrolysis of nepafenac is much greater in the retina/choroid (approximately 20 times) than in the iris/ciliary body. Finally, the administration of nepafenac also produces sustained inhibition of prostaglandin synthesis relative to a conventional NSAID. The increased absorption, targeted activation, and greater duration of action of nepafenac may lead to improved efficacy in the posterior segment over other NSAIDs lacking these properties. ${ }^{33}$ In a rabbit model of retinal inflammation, topical nepafenac readily penetrated into the posterior segment, where it decreased prostaglandin E2 concentrations and inhibited the breakdown of the blood-retinal barrier. Other NSAIDs, including ketorolac and diclofenac, failed to inhibit accumulation of these inflammatory markers. ${ }^{34}$

Varying results are found in literature comparing efficacy of different NSAIDs for prevention of PCME. In a prospective, randomized trial on 200 eyes comparing the effect of $0.1 \%$ nepafenac and $0.4 \%$ ketorolac tromethamine in patients with low risk of PCME, the overall incidence of subclinical PCME was found as $22.7 \%$ and macular thickening was similar with 2 drugs. ${ }^{15}$ Another study compared a fixed combination of dexamethasone-netilmicin with additional NSAIDs (bromfenac, nepafenac, indomethacin, or diclofenac). CFT at 1 week decreased only in the group treated with nepafenac $(-1.3 \%, p=0.04)$, increased in fixed combination $(+4.3 \%, p=0.04)$, and did not change in bromfenac $(-1.1 \%, p=0.30)$, indomethacin $(+0.1 \%, p=0.19)$, or diclofenac $(+1.2 \%, p=0.74) .{ }^{35}$ Studies measuring prostaglandins in aqueous showed minimum levels of prostaglandins in patients given preoperatively nepafenac compared to ketorolac and bromefenac. Thus, topical use of nepafenac has been recommended for preventing PCME in patients with NPDR. ${ }^{17}$ Nepafenac is also effective in the treatment of PCME including chronic cases which had failed to respond to conventional NSAID/steroid therapy. ${ }^{26,33}$ 


\section{Topical steroids}

The beneficial effect of PA eye drops after cataract surgery has never been described; rather, the use of PA has remained a legacy of practice for over 50 years. ${ }^{11}$ Corticosteroids are effective in suppressing postoperative inflammation, but have little suppression effect on PCME and potentially increase intraocular pressure. In several studies, almost all PCME cases occurred in spite of conventional postoperative topical steroid treatment including PA or dexamethasone for 4-6 weeks., ${ }^{2,8,16}$ The problems with steroids are limited penetration and adverse effects with prolonged use like intraocular pressure spikes, delayed wound healing, and herpes virus activation. ${ }^{30}$ Due to anatomic membrane barriers and the lachrymal drainage, it can be difficult to obtain therapeutic drug concentrations in the posterior parts of the eye after topical administration. Invasive methods such as direct steroid injection into the vitreous and subtenons capsule may be necessary. ${ }^{36}$ With most formulations, only a small fraction of an eye drop dose will reach the posterior segment after topical administration ( $<5 \%$ in general) via transcorneal permeation or by permeation through conjunctiva and sclera. A fraction of $50 \%-95 \%$ is absorbed systematically through the conjunctiva or nose. Unless specific formulations like dexamethasone-cyclodextrin combination are used, it is hard to deliver significant levels of dexamethasone to the retina and vitreous in the rabbit eye. ${ }^{37}$ PCME was reported to be 6-7 times more prevalent in patients receiving topical steroids, compared with topical NSAIDs, 4-5 weeks after cataract surgery..$^{10}$ Even subconjunctival injection of triamcinolone was found less effective in PCME prevention compared to topical NSAIDs. ${ }^{11}$ While topical and subconjunctival steroids are not so effective in the prevention of PCME, intravitreal dexamethasone implant can be effective in the treatment of PCME. These facts indicate the importance of drug delivery to the posterior segment of the eye.

\section{PCME in patients with DR}

Modern cataract surgery, characterized by small incisions and short operating times, has little influence on the progression of DR, except on developing ME. ${ }^{10}$ Development of ME after cataract surgery in diabetics depends on the duration, severity, and control of DM, along with hypertension, preexisting ME, prior treatment with lasers, intravitreal injections, DR, and operative complications. Eyes with DR are associated with a 2- to 3-fold increased risk of ME. ${ }^{11,17}$ Chen et al reported the incidence of ME after cataract surgery as $22.8 \%$ in diabetic eyes. ${ }^{20} \mathrm{~A}$ higher incidence of ME after cataract surgery is reported in eyes with DR, and worsening of ME often occurs after surgery in eyes with preoperative DME. ${ }^{2,20}$
An increased level of inflammatory mediators has been found in the vitreous and aqueous humor in patients with DR causing subclinical intraocular inflammation, which may worsen after cataract surgery. This may explain the significantly higher incidence of PCME in patients with DR. ${ }^{10}$ Postoperative ME may be due to the development of PCME, worsening of DME, or combination of both. ${ }^{4}$

In Singh et al's industry-supported, multicenter, randomized, double-masked, vehicle-controlled study including 263 adult diabetics with NPDR requiring cataract surgery, patients were randomized (1:1) to instill nepafenac $0.1 \%$ or vehicle 3 times daily beginning 1 day prior to surgery through day 90 . The assessment of efficacy included the percentage of patients who developed ME ( $\geq 30 \%$ increase in central subfield macular thickness from baseline). A significantly lower percentage of patients in the nepafenac group developed ME compared to vehicle group $(3.2 \%$ versus $16.7 \% ; p<0.001)$. There was no clinically relevant increase in risk from 90 days dosing compared with 14 days. Therefore, given the benefits in preventing PCME and maintaining vision, the risk/benefit ratio of nepafenac $0.1 \%$ prophylaxis in patients with NPDR was found positive. ${ }^{2}$ In Sarfraz et al's nonindustry-supported, prospective study including 60 patients with NPDR undergoing phacoemulsification, Group 1 received $0.1 \%$ nepafenac after cataract surgery for 3 months, along with routine postoperative medications, and Group 2 received only routine postoperative medications. PCME occurred in $3.3 \%$ in nepafenac group and $23.3 \%$ in group treated with routine postoperative medications. The difference of mean change in central macular thickness between groups was statistically significant $(p<0.05)$. They concluded that $0.1 \%$ nepafenac was effective in the prevention of PCME in patients with NPDR. They added NPDR was a more important risk factor for the development of PCME than DM itself. ${ }^{17} \mathrm{~A}$ randomized, double-masked, vehicle-controlled, parallel-group study conducted at 32 centers across the world compared nepafenac $0.1 \%$ with vehicle 3 times daily. A significantly less incidence of PCME was reported in nepafenac group $(5.0 \%)$ compared to vehicle $(17.5 \%, p=0.01)$. Visual recovery was better in nepafenac, although it did not show statistical significance $(p=0.14)$. Nepafenac prevented PCME after cataract surgery in patients with DR in this study group. ${ }^{16}$ All these studies bring strong evidence which may convince even the most skeptic authors.

\section{Nepafenac $0.1 \%$ and nepafenac $0.3 \%$}

Nepafenac ophthalmic suspensions, $0.1 \%$ and $0.3 \%$, are topical NSAID products approved in the US, Europe, and various other countries to treat pain and inflammation associated 
with cataract surgery. Nepafenac $0.1 \%$ is also approved in Europe for the reduction in the risk of PCME associated with cataract surgery in diabetic patients. In the prevention of PCME, nepafenac $0.1 \%$ is usually started at 1 day before surgery, and continued on the day of surgery (30-120 minutes prior to surgery) and for 90 days thereafter. ${ }^{2}$ While nepafenac $0.1 \%$ is used 3 times a day, nepafenac $0.3 \%$ is used once daily. In multiple-dosed rabbit eyes, with $0.3 \%$ nepafenac instilled once daily or $0.1 \%$ nepafenac instilled 3 times daily, cumulative 24-hour locally distributed levels of nepafenac in posterior pole retina sub-samples were similar between these groups. ${ }^{32}$ Nonclinical and clinical studies have shown that nepafenac and amfenac reach the posterior segment of the eye after topical administration. Both nepafenac and its active metabolite amfenac block the inflammation-mediated breakdown of the blood-retinal barrier that contributes to plasma extravasation and edema. ${ }^{7}$ Nepafenac $0.3 \%$ has a particle size approximately $40 \%$ smaller than nepafenac $0.1 \%$ and delivers twice the drug concentration in the target tissue compared to nepafenac $0.1 \%$.

Most of the studies published in the literature have been conducted using nepafenac $0.1 \%$. There are few studies on the efficacy of $0.3 \%$ nepafenac formulation. In a Phase III study by Modi et al, once-daily nepafenac $0.3 \%$ was found noninferior to nepafenac $0.1 \% 3$ times daily for the prevention and treatment of ocular inflammation after cataract surgery and both formulations were superior to vehicle $(p<0.0001)$. The safety of nepafenac $0.3 \%$ was comparable to that of nepafenac $0.1 \%$, with the added convenience of once-daily dosing. ${ }^{38}$ In 2 parallel, randomized, vehicle-controlled Phase III studies, Singh et al demonstrated that nepafenac $0.3 \%$, when given once daily beginning 1 day before surgery and continued for 90 days, was superior to vehicle in reducing the risk of ME after cataract surgery in patients with DM. Significantly lower percentage of patients demonstrated ME within 90 days after surgery with nepafenac $0.3 \%$ versus vehicle $(2.3 \%$ versus $17.3 \% ; p<0.001)$. Furthermore, a higher percentage of patients in the nepafenac group compared with the vehicle group had improvements of 15 Early Treatment DR Study (ETDRS) letters. ${ }^{7}$

In McCafferty et al's study, 1,000 eyes were randomized to placebo (497) or nepafenac $0.3 \%$ (503) used once daily, postoperatively for 5 weeks at 2 ophthalmology clinics. All patients received topical prednisolone 1\% 4 times daily for the first week, tapered to 2 times daily over the second week, and 1 time daily for the subsequent 3 weeks which approximates the most common practice. The incidence of PCME was $4.2 \%$ in patients with different risk factors
(DR, contralateral PCME, etc) and 2.0\% without risk factors. Topical nepafenac $0.3 \%$ significantly reduced the incidence of PCME compared to placebo when used after routine cataract surgery $(p=0.0001)$. When patients with preoperative risk factors were excluded, the incidence of PCME between treatment and placebo groups was equivalent $(p=0.31)$. They concluded that topical nepafenac $0.3 \%$ reduces PCME in patients with preoperative risk factors for PCME compared to placebo but shows no benefit in patients without preoperative risk factors. ${ }^{8}$ The use of topical nepafenac in adjunction with topical steroids is recommended for prevention of ME in patients with NPDR. ${ }^{17}$ Furthermore, in Singh et al's study, a significantly greater percentage of patients in the nepafenac group than in the vehicle group showed improvements of $\geq 15$ ETDRS letters from the postsurgical baseline to day $90(56.8 \%$ versus $41.9 \%$; $p=0.019)$. This study supports the beneficial effects of topical nepafenac $0.1 \%$ in the prevention of PCME and associated visual loss. ${ }^{2}$

\section{Safety}

Because of the class effects for NSAIDs, corneal thinning, erosion, delayed wound healing, ulceration, and perforation may occur with nepafenac use in patients with compromised corneas. Patients with surgically cut corneal nerves, penetrating grafts, dry eye syndrome, and rheumatoid arthritis are not good candidates for nepafenac use. In Singh et al's study including high-risk patients with DM, a few patients experienced adverse effects. The overall safety profile of nepafenac $0.3 \%$, dosed once daily beginning the day before surgery and continued for 90 days thereafter, was comparable with that of nepafenac $0.1 \%$ dosed thrice daily for the same duration in patients with DR. ${ }^{2,7}$ In Shorstein et al's study, no instances of cornea melt was observed and postoperative corneal problems occurred in patients using PA and NSAID (3 in 4,754 eyes; 0.06\%). ${ }^{11}$ No safety issues or trends were identified when dosing was increased to 90 days that negatively impacted the favorable benefit/risk profile of nepafenac. Punctuate keratitis and corneal epithelium defect may occur in exceptional cases. Nepafenac is not recommended in patients with severe dry eye showing corneal staining. ${ }^{2}$ In Pollack et al's study, a 90-day nepafenac $0.1 \%$ treatment after cataract surgery in patients with DR demonstrated no safety issues. ${ }^{16}$

\section{Conclusion}

Results of recent studies suggest that nepafenac PCME prophylaxis is unnecessary in patients without risk factors. ${ }^{8}$ Therefore, it is necessary to select eyes with high risk factors 
for PCME and treat them efficiently. ${ }^{13}$ Although there are some contrary reports, most authors consider DM as a risk factor for the development of PCME because of the breakdown of blood-retinal barrier secondary to diabetic vasculopathy. ${ }^{2,13}$ Severity of PCME depends on the duration, severity, and control of DM, along with hypertension. Presence of DR is associated with a 2- to 3-fold increased risk of PCME. Complicated surgery increases the risk even further. Hence, patients with DM, especially those with longstanding advanced DM, and DR should be treated for PCME. Once-daily dosing regimen of nepafenac $0.3 \%$ may improve postoperative outcomes with increased patient compliance and reduced treatment burden. In healthy individuals without DM, PCME history in the other eye may be an indication for nepafenac prophylaxis. However, each patient should be assessed individually in terms of benefits and adverse effects of the treatment.

\section{Disclosure}

The authors report no conflicts of interest in this work. The authors alone are responsible for the content and writing of the paper.

\section{References}

1. Shields MK, Adler PA, Fuzzard DR, Chalasani R, Teong JM. A case of acute bilateral Irvine-Gass syndrome following uncomplicated phacoemulsification, demonstrated with optical coherence tomography. Case Rep Ophthalmol. 2015;6(1):143-148.

2. Singh R, Alpern L, Jaffe GJ, et al. Evaluation of nepafenac in prevention of macular edema following cataract surgery in patients with diabetic retinopathy. Clin Ophthalmol. 2012;6:1259-1269.

3. Irvine SR. A newly defined vitreous syndrome following cataract surgery. Am J Ophthalmol. 1953;36(5):599-619.

4. Munk MR, Jampol LM, Simader C, et al. Differentiation of diabetic macular edema from pseudophakic cystoid macular edema by spectraldomain optical coherence tomography. Invest Ophthalmol Vis Sci. 2015; 56(11):6724-6733.

5. Chu CJ, Johnston RL, Buscombe C, Sallam AB, Mohamed Q, Yang YC; United Kingdom Pseudophakic Macular Edema Study Group. Risk factors and incidence of macular edema after cataract surgery: a database study of 81984 eyes. Ophthalmology. 2016;123(2):316-323.

6. Garcia JM, Isaac DL, Ávila MP. Dexamethasone $0.7 \mathrm{mg}$ implants in the management of pseudophakic cystoid macular edema. Arq Bras Oftalmol. 2016;79(2):113-115.

7. Singh RP, Lehmann R, Martel J, et al. Nepafenac $0.3 \%$ after cataract surgery in patients with diabetic retinopathy: results of 2 randomized Phase 3 studies. Ophthalmology. 2017;124(6):776-785.

8. McCafferty S, Harris A, Kew C, et al. Pseudophakic cystoid macular edema prevention and risk factors; prospective study with adjunctive once daily topical nepafenac $0.3 \%$ versus placebo. BMC Ophthalmol. 2017;17(1):16

9. Wielders LH, Schouten JS, Aberle MR, et al. Treatment of cystoid macular edema after cataract surgery. J Cataract Refract Surg. 2017; 43(2):276-284.

10. Medić A, Jukić T, Matas A, Vukojević K, Sapunar A, Znaor L. Effect of preoperative topical diclofenac on intraocular interleukin-12 concentration and macular edema after cataract surgery in patients with diabetic retinopathy: a randomized controlled trial. Croat Med J. 2017;58(1):49-55.
11. Shorstein NH, Liu L, Waxman MD, Herrinton LJ. Comparative effectiveness of three prophylactic strategies to prevent clinical macular edema after phacoemulsification surgery. Ophthalmology. 2015; 122(12):2450-2456.

12. Makri OE, Tsapardoni FN, Plotas P, Ifantis N, Xanthopoulou PT, Georgakopoulos CD. Cystoid macular edema associated with preservative-free latanoprost after uncomplicated cataract surgery: case report and review of the literature. BMC Res Notes. 2017;10(1):127.

13. Do JR, Oh JH, Chuck RS, Park CY. Transient corneal edema is a predictive factor for pseudophakic cystoid macular edema after uncomplicated cataract surgery. Korean J Ophthalmol. 2015;29(1):14-22.

14. Yang J, Cai L, Sun Z, et al. Risk factors for and diagnosis of pseudophakic cystoid macular edema after cataract surgery in diabetic patients. J Cataract Refract Surg. 2017;43(2):207-214.

15. Ramakrishnan S, Baskaran P, Talwar B, Venkatesh R. Prospective, randomized study comparing the effect of $0.1 \%$ nepafenac and $0.4 \%$ ketorolac tromethamine on macular thickness in cataract surgery patients with low risk for cystoid macular edema. Asia Pac J Ophthalmol (Phila). 2015;4(4):216-220.

16. Pollack A, Staurenghi G, Sager D, Mukesh B, Reiser H, Singh RP. Prospective randomised clinical trial to evaluate the safety and efficacy of nepafenac $0.1 \%$ treatment for the prevention of macular oedema associated with cataract surgery in patients with diabetic retinopathy. Br J Ophthalmol. 2017;101(4):423-427.

17. Sarfraz MH, Haq RI, Mehboob MA. Effect of topical nepafenac in prevention of macular edema after cataract surgery in patients with nonproliferative diabetic retinopathy. PakJ Med Sci. 2017;33(1):210-214.

18. Gekka M, Miyata K, Nagai Y, et al. Corneal epithelial barrier function in diabetic patients. Cornea. 2004;23(1):35-37.

19. Saini JS, Mittal S. In vivo assessment of corneal endothelial function in diabetes mellitus. Arch Ophthalmol. 1996;114(6):649-653.

20. Chen XY, Song WJ, Cai HY, Zhao L. Macular edema after cataract surgery in diabetic eyes evaluated by optical coherence tomography. Int J Ophthalmol. 2016;9(1):81-85.

21. Schmier JK, Covert DW, Hulme-Lowe CK, Mullins A, Mahlis EM. Treatment costs of cystoid macular edema among patients following cataract surgery. Clin Ophthalmol. 2016;10:477-483.

22. Tzelikis P, Vieira M, Alves M, et al. Comparison of ketorolac $0.4 \%$ and nepafenac $0.1 \%$ for the prevention of cystoid macular oedema after phacoemulsification: prospective placebo-controlled randomised study. Br J Ophthalmol. 2015;99(5):654-658.

23. Almeida DR, Khan Z, Xing L, et al. Prophylactic nepafenac and ketorolac versus placebo in preventing postoperative macular edema after uneventful phacoemulsification. J Cataract Refract Surg. 2012;38(9): $1537-1543$.

24. Mathys KC, Cohen KL. Impact of nepafenac $0.1 \%$ on macular thickness and postoperative visual acuity after cataract surgery in patients at low risk for cystoid macular oedema. Eye (Lond). 2010;24(1):90-96.

25. Tsilimbaris MK, Tsika C, Kymionis GD. Intravitreal ketorolac for the treatment of chronic cystoid macular edema after cataract surgery. Ther Clin Risk Manag. 2016;12:177-182.

26. Yüksel B, Uzunel UD, Kerci SG, Sagban L, Kusbeci T, Orsel T. Comparison of subtenon triamcinolone acetonide injection with topical nepafenac for the treatment of pseudophakic cystoid macular edema. Ocul Immunol Inflamm. 2016;25:1-7.

27. Boscia F, Giancipoli E, D’Amico Ricci G, Pinna A. Management of macular oedema in diabetic patients undergoing cataract surgery. Curr Opin Ophthalmol. 2017;28(1):23-28.

28. Lim BX, Lim CH, Lim DK, Evans JR, Bunce C, Wormald R. Prophylactic non-steroidal anti-inflammatory drugs for the prevention of macular oedema after cataract surgery. Cochrane Database Syst Rev. 2016;11: CD006683.

29. Modjtahedi BS, Paschal JF, Batech M, Luong TQ, Fong DS. Perioperative topical nonsteroidal anti-inflammatory drugs for macular edema prophylaxis following cataract surgery. Am J Ophthalmol. 2017;176: 174-182. 
30. Kim SJ, Schoenberger SD, Thorne JE, Ehlers JP, Yeh S, Bakri SJ. Topical nonsteroidal anti-inflammatory drugs and cataract surgery: a report by the American Academy of Ophthalmology. Ophthalmology. 2015; 122(11):2159-2168.

31. Kessel L, Tendal B, Jørgensen KJ, et al. Post-cataract prevention of inflammation and macular edema by steroid and nonsteroidal antiinflammatory eye drops: a systematic review. Ophthalmology. 2014; 121(10):1915-1924.

32. Chastain JE, Sanders ME, Curtis MA, et al. Distribution of topical ocular nepafenac and its active metabolite amfenac to the posterior segment of the eye. Exp Eye Res. 2016;145:58-67.

33. Hariprasad SM, Callanan D, Gainey S, He YG, Warren K. Cystoid and diabetic macular edema treated with nepafenac $0.1 \%$.J Ocul Pharmacol Ther. 2007;23(6):585-590.

34. Kapin MA, Yanni JM, Brady MT, et al. Inflammation-mediated retinal edema in the rabbit is inhibited by topical nepafenac. Inflammation. 2003;27(5):281-291.
35. Cardascia N, Palmisano C, Centoducati T, Alessio G. Topical nonsteroidal anti-inflammatory drugs as adjuvant therapy in the prevention of macular edema after cataract surgery. Int Ophthalmol. Epub 2016 Oct 20 .

36. Loftsson T, Sigurdsson HH, Konrádsdóttir F, Gísladóttir S, Jansook P, Stefánsson E. Topical drug delivery to the posterior segment of the eye: anatomical and physiological considerations. Pharmazie. 2008;63(3): 171-179.

37. Sigurdsson HH, Konráethsdóttir F, Loftsson T, Stefánsson E. Topical and systemic absorption in delivery of dexamethasone to the anterior and posterior segments of the eye. Acta Ophthalmol Scand. 2007;85(6): 598-602.

38. Modi SS, Lehmann RP, Walters TR, et al. Once-daily nepafenac ophthalmic suspension $0.3 \%$ to prevent and treat ocular inflammation and pain after cataract surgery: Phase 3 study. J Cataract Refract Surg. 2014;40(2):203-211.
Clinical Ophthalmology

\section{Publish your work in this journal}

Clinical Ophthalmology is an international, peer-reviewed journal covering all subspecialties within ophthalmology. Key topics include: Optometry; Visual science; Pharmacology and drug therapy in eye diseases; Basic Sciences; Primary and Secondary eye care; Patient Safety and Quality of Care Improvements. This journal is indexed on

\section{Dovepress}

PubMed Central and CAS, and is the official journal of The Society of Clinical Ophthalmology (SCO). The manuscript management system is completely online and includes a very quick and fair peer-review system, which is all easy to use. Visit http://www.dovepress.com/ testimonials.php to read real quotes from published authors. 